\title{
Cut Bogner-Fox-Schmit elements for plates
}

\author{
Erik Burman ${ }^{1}$, Peter Hansbo ${ }^{2^{*}}$ (D) and Mats G. Larson ${ }^{3}$
}

*Correspondence:

Peter.Hansbo@ju.se

${ }^{2}$ Department of Mechanical

Engineering, Jönköping

University, 55111 Jönköping,

Sweden

Full list of author information is

available at the end of the article

\begin{abstract}
We present and analyze a method for thin plates based on cut Bogner-Fox-Schmit elements, which are $C^{1}$ elements obtained by taking tensor products of Hermite splines. The formulation is based on Nitsche's method for weak enforcement of essential boundary conditions together with addition of certain stabilization terms that enable us to establish coercivity and stability of the resulting system of linear equations. We also take geometric approximation of the boundary into account and we focus our presentation on the simply supported boundary conditions which is the most sensitive case for geometric approximation of the boundary.
\end{abstract}

Keywords: Kirchhoff plate, Cut finite element method, Rectangular plate element

\section{Introduction}

The Bogner-Fox-Schmit (BFS) element [6] is a classical $C^{1}$ thin plate element obtained by taking tensor products of cubic Hermite splines and removing the interior degrees of freedom that are zero on the boundary. In this paper, we consider a variant where we retain these degrees of freedom to obtain a $C^{1}$ version of the $Q_{3}$ approximation [23]. This element is only $C^{1}$ on tensor product (rectangular) elements, which is a serious drawback since it severely limits the applicability of the resulting finite element method. However, on geometries allowing for tensor product discretization it is generally considered to be one of the most efficient elements for plate analysis, cf. [24, p. 153]. It is also a reasonably low order element for plates which is very simple to implement, in contrast with triangular elements which either use higher order polynomials, such as the Argyris element [1], or macro element techniques, such as the Clough-Tocher element [10]. The construction of curved versions of these elements for boundary fitting can also be cumbersome, see, e.g., [5]. It should be noted that the use of straight line segments for discretizing the boundary is not to be recommended, not only because of accuracy issues but also due to Babuška's paradox for simply supported plates, see [3].

An alternative to $C^{1}$ approximations for Kirchhoff plates is to either use discontinuous Galerkin methods $[8,15,17,22]$, or to use mixed finite elements for the Reissner-Mindlin model with small plate thickness $[2,4,12]$. These $C^{0}$ methods alleviate the problem of boundary approximation. In this paper we present an alternative idea where $C^{1}$ continuity is retained: we develop a cut finite element version, allowing for discretizing a smooth boundary which may cut through the tensor product mesh in an arbitrary manner. Adding stabilization terms on the faces associated with elements that intersect the boundary, we

(c) The Author(s) 2020. This article is licensed under a Creative Commons Attribution 4.0 International License, which permits use, sharing, adaptation, distribution and reproduction in any medium or format, as long as you give appropriate credit to the original author(s) and the source, provide a link to the Creative Commons licence, and indicate if changes were made. The images or other third party material in this article are included in the article's Creative Commons licence, unless indicated otherwise in a credit line to the material. If material is not included in the article's Creative Commons licence and your intended use is not permitted by statutory regulation or exceeds the permitted use, you will need to obtain permission directly from the copyright holder. To view a copy of this licence, visit http://creativecommons.org/licenses/by/4.0/. 
obtain a stable method with optimal order convergence. We prove a priori error estimates which also take approximation of the boundary into account. The focus of the analysis is on simply supported boundary conditions, the computationally most challenging case.

The paper is organized as follows. In "The Kirchhoff plate" section, we recall the thin plate Kirchhoff model; in "The finite element method" section we formulate the cut finite element method; in "Error estimates" section we present the analysis of the method starting with a sequence of technical results leading up to a Strang Lemma and an estimate of the consistency error and finally a priori error estimates in the energy and $L^{2}$ norms. In "Numerics" section, we present some numerical illustrations, and in "Concluding remarks" section some concluding remarks are included.

\section{The Kirchhoff plate}

Consider a simply supported thin plate in a domain $\Omega \subset \mathbb{R}^{2}$ with smooth boundary $\partial \Omega$. The displacement $u: \Omega \rightarrow \mathbb{R}$ satisfies

$$
\nabla \cdot(\sigma(\nabla u) \cdot \nabla)=f
$$

where $\sigma(\nabla v)$ is the stress tensor

$$
\sigma(\nabla v)=\kappa\left(\epsilon(\nabla v)+v(1-v)^{-1}(\nabla \cdot(\nabla v)) I=\kappa\left(\nabla \otimes \nabla v+v(1-v)^{-1}(\Delta v) I\right)\right)
$$

with $\epsilon(\nabla v)$ the strain tensor

$$
\epsilon(\nabla v)=((\nabla v) \otimes \nabla+\nabla \otimes(\nabla v)) / 2=\nabla \otimes \nabla v
$$

and $\kappa$ the parameter

$$
\kappa=\frac{E t^{3}}{12(1+v)}
$$

with $E$ the Young's modulus, $v$ the Poisson's ratio, and $t$ the plate thickness. Since $0 \leq$ $v \leq 0.5$ both $\kappa$ and $v(1-v)^{-1}$ are uniformly bounded.

We shall focus our presentation on simply supported boundary conditions

$$
u=0 \quad \text { on } \partial \Omega, \quad M_{n n}(u)=0 \quad \text { on } \partial \Omega
$$

where the moment tensor $M$ is defined by

$$
M(u)=\sigma(\nabla u)
$$

and $M_{a b}=a \cdot M \cdot b$ for $a, b \in \mathbb{R}^{2}$. Other conditions, such as clamped boundaries, can be handled using the same techniques as in the following, cf. [15].

The weak form of (1) and (5) takes the form: find $u \in V=\left\{v \in H^{2}(\Omega): v=0\right.$ on $\left.\partial \Omega\right\}$ such that

$$
a(u, v)=l(v) \quad \forall v \in V
$$

where

$$
\left.a(v, w)=(\sigma(\nabla v), \epsilon(\nabla w))_{\Omega}=\kappa(\nabla \otimes \nabla v, \nabla \otimes \nabla w)_{\Omega}+v(1-v)^{-1}(\Delta v, \Delta w)_{\Omega}\right)
$$

and $l(v)=(f, v)_{\Omega}$. The form $a$ is symmetric, continuous, and coercive on $V$ equipped with the $H^{2}(\Omega)$ norm and it follows from the Lax-Milgram theorem that there exists a unique solution in $V$ to (7). Furthermore, for smooth boundary and $f \in L^{2}$ we have the elliptic regularity

$$
\|u\|_{H^{4}(\Omega)} \lesssim\|f\|_{\Omega}
$$




\section{The finite element method}

\section{The mesh and finite element space}

We begin by introducing the following notation.

- Let $\widetilde{\mathcal{T}}_{h}, h \in\left(0, h_{0}\right]$, be a family of partitions of $\mathbb{R}^{2}$ into squares with side $h$. Let $\widetilde{V}_{h}$ be the Bogner-Fox-Schmit space consisting of tensor products of cubic Hermite splines on $\widetilde{\mathcal{T}}_{h}$. Note that $\left.\widetilde{V}_{h}\right|_{T}=Q_{3}(T)$, with $Q_{3}(T)$ the tensor product $P_{3}\left(I_{1}\right) \otimes P_{3}\left(I_{2}\right)$ of cubic polynomials where $T=I_{1} \times I_{2} \subset \mathbb{R}^{2}$.

- Let $\rho$ be the signed distance function, positive on the outside and negative on the inside, associated with $\partial \Omega$ and let $U_{\delta}(\partial \Omega)=\left\{x \in \mathbb{R}^{2}:|\rho(x)|<\delta\right\}$ be the tubular neighborhood of $\partial \Omega$ of thickness $2 \delta$. Then there is $\delta_{0}>0$ such that the closest point mapping $p: U_{\delta_{0}}(\partial \Omega) \rightarrow \partial \Omega$ is a well defined function of the form $p(x)=$ $x-\rho(x) n(p(x))$, cf. [14, Section 14.6].

- Let $\left\{\Omega_{h}, h \in\left(0, h_{0}\right]\right\}$ be a family of approximations of $\Omega$ such that $\partial \Omega_{h} \subset U_{\delta_{0}}(\partial \Omega)$ is piecewise smooth and

$$
\begin{aligned}
\|\rho\|_{L^{\infty}\left(\partial \Omega_{h}\right)} & \lesssim h^{4} \\
\left\|n(p)-n_{h}\right\|_{L^{\infty}\left(\partial \Omega_{h}\right)} & \lesssim h^{3}
\end{aligned}
$$

Furthermore, we assume that for each element $T$ such that $\partial \Omega_{h}$ intersects the interior of $T$, i.e. $\operatorname{int}(T) \cap \partial \Omega_{h} \neq \emptyset$, the curve segment $\partial \Omega_{h} \cap T$ is smooth and intersect the boundary $\partial T$ of $T$ in precisely two different points. Let $\mathcal{X}_{h}$ be the set of all points where $\partial \Omega_{h}$ is not smooth and note that the number of elements $\left|\mathcal{X}_{h}\right|$ in $\mathcal{X}_{h}$ satisfies $\left|\mathcal{X}_{h}\right| \lesssim h^{-1}$.

- Let $\mathcal{T}_{h}=\left\{T \in \widetilde{\mathcal{T}}_{h}: T \cap \Omega_{h} \neq \emptyset\right\}$ be the active mesh and $\mathcal{F}_{h}$ the set of interior faces in $\mid \mathcal{T}_{h}$. Let $\mathcal{T}_{h, I}$ be the set of elements such that $T \subset \Omega \cap \Omega_{h}$ and let $\mathcal{F}_{h, I}$ be the set of interior faces in $\mathcal{T}_{h, I}$. Let $\mathcal{T}_{h, B}=\mathcal{T}_{h} \backslash \mathcal{T}_{h, I}$ and $\mathcal{F}_{h, B}=\mathcal{F}_{h} \backslash \mathcal{F}_{h, I}$. For simplicity we assume that

$$
\Omega \subset O_{h}:=\cup_{T \in \mathcal{T}_{h}} T
$$

We can always satisfy this assumption by enlarging the active mesh $\mathcal{T}_{h}$ if necessary.

- Let $V_{h}$ be the restriction of $\widetilde{V}_{h}$ to $\mathcal{T}_{h}$. Let $\mathcal{K}_{h}=\mathcal{T}_{h} \cap \Omega_{h}$ be the intersection of the active elements $T$ with $\Omega_{h}$.

We illustrate some of these quantities in Fig. 1. Here, $\partial \Omega_{h}$ is indicated by the dotted line; $\mathcal{T}_{h}$ is the union of all elements shown; $\mathcal{T}_{h, I}$ consists of the white elements and $\mathcal{T}_{h, B}$ the grey elements. $\mathcal{K}_{h}$ consists of the elements and cut parts of elements inside $\partial \Omega_{h}$ and $\mathcal{F}_{h, B}$ consists of all element sides on the grey elements excluding those without neighbouring elements.

\section{The finite element method}

The method reads: find $u_{h} \in V_{h}$ such that

$$
A_{h}\left(u_{h}, v\right)=l_{h}(v) \quad \forall v \in V_{h}
$$

The forms are defined by

$$
\begin{aligned}
& A_{h}(v, w)=a_{h}(v, w)+\beta s_{h}(v, w) \\
& a_{h}(v, w)=(\sigma(\nabla v), \epsilon(\nabla w))_{\Omega_{h}}+(T(v), w)_{\partial \Omega_{h}}+(v, T(w))_{\partial \Omega_{h}}
\end{aligned}
$$




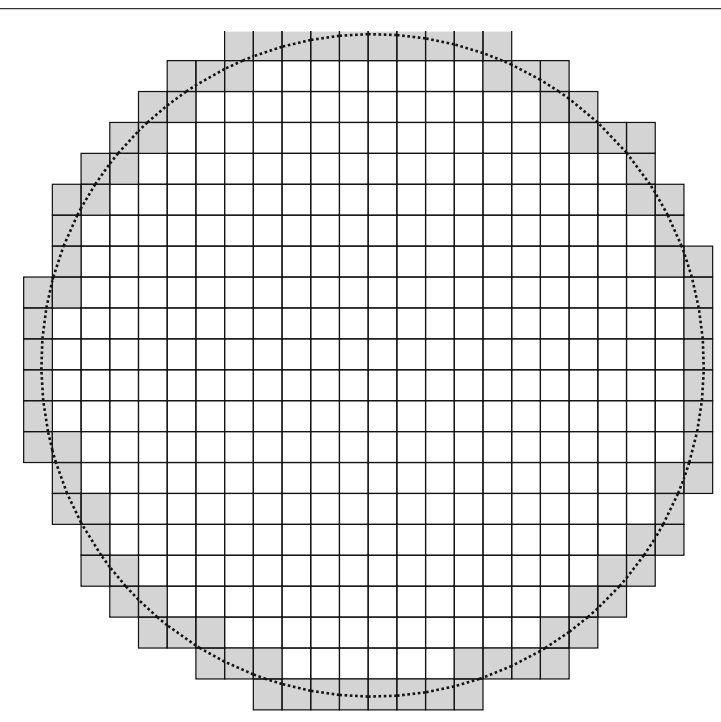

Fig. 1 Illustration of mesh definitions

$$
\begin{aligned}
& +\gamma h^{-3}(v, w)_{\partial \Omega_{h}} \\
s_{h}(v, w)= & h\left(\left[\nabla_{n_{F}}^{2} v\right],\left[\nabla_{n_{F}}^{2} w\right]\right)_{\mathcal{F}_{h, B}}+h^{3}\left(\left[\nabla_{h_{F}}^{3} v\right],\left[\nabla_{h_{F}}^{3} w\right]\right)_{\mathcal{F}_{h, B}} \\
l_{h}(v)= & (f, v)_{\Omega_{h}}
\end{aligned}
$$

where

$$
T=(M \cdot \nabla)_{n}+\nabla_{t} M_{n t}
$$

with sub-indices $n$ and $t$ indicating scalar product with the normal and tangent of $\partial \Omega_{h}$, and $\beta, \gamma$ are positive parameters which are proportional to $\kappa$. Here $s_{h}$ is a stabilization form involving jumps of the second and third normal derivatives in the direction $n_{F}$, the normal to the element face, with

$$
\left.[v]:=\lim _{\epsilon \downarrow 0}\left(v\left(x+\epsilon n_{F}\right)-v\left(x-\epsilon n_{F}\right)\right)\right)
$$

which provides necessary stability at the cut elements, see (23). The bilinear form, apart from the stabilization terms, stems from Nitsche's method [20], first analyzed for plates in a discontinuous Galerkin setting in [15].

\section{Error estimates}

\section{Basic properties of $A_{h}$}

\section{The energy norm}

Define the following energy norm on $V+V_{h}$, with $V=H^{4}\left(O_{h}\right)$,

$$
\|v\|_{h}^{2}=\|v\|_{\Omega_{h}}^{2}+\beta\|v\|_{s_{h}}^{2}+h^{3}\|T(v)\|_{\partial \Omega_{h}}^{2}+h^{-3}\|v\|_{\partial \Omega_{h}}^{2}
$$

where

$$
\|v\|_{\Omega_{h}}^{2}=(\sigma(\nabla v), \epsilon(\nabla v))_{\Omega_{h}}
$$

and we employ the standard notation $\|v\|_{s_{h}}^{2}=s_{h}(v, v)$. In view of (8) we have $\kappa\left\|\nabla^{2} v\right\|_{\Omega_{h}}^{2} \lesssim$ $\|v\|_{\Omega_{h}}^{2}$, where $\nabla^{j} v$ is the tensor of all $j:$ th order derivatives. 


\section{Stabilization}

The stabilization term provides us with the following bound

$$
\left\|\nabla^{j} v\right\|_{\mathcal{T}_{h}}^{2} \lesssim\left\|\nabla^{j} v\right\|_{\mathcal{T}_{h, I}}+h^{2(2-j)}\|v\|_{s_{h}}^{2}, \quad j=0,1,2,3
$$

which follows from the standard estimate

$$
\left\|\nabla^{j} v\right\|_{T_{2}}^{2} \lesssim\left\|\nabla^{j} v\right\|_{T_{1}}^{2}+\sum_{k=j}^{p} h^{2(k-j)}\left\|\left[\nabla^{k} v\right]\right\|_{F}^{2}
$$

where $T_{1}$ and $T_{2}$ are elements that share the face $F$, and $\left.v\right|_{T_{i}} \in P_{p}\left(T_{i}\right)$, the space of polynomials of order $p$. See for instance $[16,19]$ for further details.

\section{Continuity and coercivity}

The form $A_{h}$ is continuous

$$
A_{h}(v, w) \lesssim\|v\|_{h}\|w\|_{h} \quad v, w \in V+V_{h}
$$

which follows directly from the Cauchy-Schwarz inequality, and for $\gamma$ large enough coercive

$$
\|v\|_{h}^{2} \lesssim A_{h}(v, v) \quad v \in V_{h}
$$

\section{Verification of (25)}

We first recall the cut trace inequality

$$
\|v\|_{\partial \Omega_{h} \cap T}^{2} \lesssim h^{-1}\|v\|_{T}^{2}+h\|\nabla v\|_{T}^{2} \quad v \in H^{1}(T)
$$

see [25] for a derivation. For $\left.v \in V_{h}\right|_{T}=V_{h}(T)$ we have the standard inverse inequality

$$
\left\|\nabla^{k} v\right\|_{T} \lesssim h^{l-k}\left\|\nabla^{l} v\right\|_{T} \quad v \in V_{h}(T), \quad k \geq l
$$

which combined with (26) give the cut inverse trace inequality

$$
\|v\|_{\partial \Omega_{h} \cap T}^{2} \lesssim h^{-1}\|v\|_{T}^{2} \quad V_{h}(T)
$$

Now, using the inverse trace inequality (28), the inverse inequality (27), followed by the stabilization estimate (22) we obtain

$$
\begin{aligned}
\kappa^{-1} h^{3}\|T(v)\|_{\partial \Omega_{h}}^{2} & \lesssim \kappa h^{2}\left\|\nabla^{3} v\right\|_{\mathcal{T}_{h}\left(\partial \Omega_{h}\right)}^{2} \lesssim \kappa\left\|\nabla^{2} v\right\|_{\mathcal{T}_{h}\left(\partial \Omega_{h}\right)}^{2} \\
& \lesssim \kappa\left(\left\|\nabla^{2} v\right\|_{\Omega_{h}}^{2}+\|v\|_{s_{h}}^{2}\right) \lesssim\|v\|_{\Omega_{h}}^{2}+\kappa\|v\|_{s_{h}}^{2}
\end{aligned}
$$

and thus there is a constant $C_{*}$ such that

$$
\kappa^{-1} h^{3}\|T(v)\|_{\partial \Omega_{h}}^{2} \leq C_{*}\left(\|v\|_{\Omega_{h}}^{2}+\kappa\|v\|_{s_{h}}^{2}\right)
$$

As a consequence, $\|v\|_{h}$ and $\|v\|_{h, *}$, where

$$
\|v\|_{h, *}^{2}=\|v\|_{\Omega_{h}}^{2}+\beta\|v\|_{s_{h}}^{2}+h^{-3}\|v\|_{\partial \Omega_{h}}^{2}
$$

are equivalent norms on $V_{h}$. We then have

$$
A_{h}(v, v)=\|v\|_{\Omega_{h}}^{2}+\beta\|v\|_{s_{h}}^{2}+2(T(v), v)_{\partial \Omega_{h}}+\gamma h^{-3}\|v\|_{\partial \Omega_{h}}^{2}
$$




$$
\begin{aligned}
& \geq\|v\|_{\Omega_{h}}^{2}+\beta\|v\|_{s_{h}}^{2}-\delta \kappa^{-1} h^{3}\|T(v)\|_{\partial \Omega_{h}}^{2}+\left(\gamma-\delta^{-1} \kappa\right) h^{-3}\|v\|_{\partial \Omega_{h}}^{2} \\
& \geq\left(1-C_{*} \delta\right)\|v\|_{\Omega_{h}}^{2}+\left(\beta-\kappa C_{*} \delta\right)\|v\|_{s_{h}}^{2}+\left(\gamma-\delta^{-1} \kappa\right) h^{-3}\|v\|_{\partial \Omega_{h}}^{2}
\end{aligned}
$$

and we find that taking $\delta$ small enough to guarantee that $1-C_{*} \delta \geq m>0, \beta$ large enough to guarantee that $\beta-\kappa C_{*} \delta \geq m$, and $\gamma$ large enough to guarantee that $\gamma-\delta^{-1} \kappa \geq m$ leads to $A_{h}(v, v) \gtrsim\|v\|_{h, *}^{2} \gtrsim\|v\|_{h}^{2}$.

\section{Poincaré inequality}

We have the following Poincaré inequality

$$
\|v\|_{H^{2}\left(\mathcal{T}_{h}\right)} \lesssim\|v\|_{h} \quad v \in V_{h}
$$

\section{Verification of (36)}

Using the stabilization estimate (22) and the fact that $\mathcal{T}_{h, I}$ is covered by $\Omega_{h}$ we have

$$
\left\|\nabla^{2} v\right\|_{O_{h}}^{2} \lesssim\left\|\nabla^{2} v\right\|_{\Omega_{h}}^{2}+\|v\|_{s_{h}}^{2} \lesssim\|v\|_{h}^{2}
$$

Next to estimate $\|v\|_{O_{h}}^{2}$ we again use the stabilization estimate (22) and the fact that $\mathcal{T}_{h, I}$ is covered by $\Omega$,

$$
\|v\|_{H^{1}\left(O_{h}\right)}^{2} \lesssim\|v\|_{H^{1}(\Omega)}^{2}+\|v\|_{s_{h}}^{2}
$$

Let $P_{1, \Omega}: L^{2}(\Omega) \rightarrow P_{1}(\Omega)$ be the $L^{2}$ projection onto the space of linear functions on $\Omega$. Then for $v \in H^{2}(\Omega)$, and in particular for $v \in V_{h} \subset H^{2}(\Omega)$, we have the Poincaré estimate

$$
\left\|v-P_{1, \Omega} v\right\|_{H^{1}(\Omega)} \lesssim\left\|\nabla^{2} v\right\|_{\Omega}
$$

and using the trace inequality $\|w\|_{\partial \Omega} \lesssim\|w\|_{H^{1}(\Omega)}$ with $w=v-P_{1, \Omega} v$, we obtain

$$
\left\|v-P_{1, \Omega} v\right\|_{\partial \Omega} \lesssim\left\|v-P_{1, \Omega} v\right\|_{H^{1}(\Omega)} \lesssim\left\|\nabla^{2} v\right\|_{\Omega}
$$

Note that the constants are independent of the mesh parameter since $\Omega$ is fixed. We then have

$$
\begin{aligned}
\|v\|_{H^{1}(\Omega)}^{2} & \lesssim\left\|\left(I-P_{1, \Omega}\right) v\right\|_{H^{1}(\Omega)}^{2}+\left\|P_{1, \Omega} v\right\|_{H^{1}(\Omega)}^{2} \\
& \lesssim\left\|\nabla^{2} v\right\|_{\Omega}^{2}+\left\|P_{1, \Omega} v\right\|_{\partial \Omega_{h}}^{2} \\
& \lesssim \underbrace{\left\|\nabla^{2} v\right\|_{\Omega_{h}}^{2}+\|v\|_{s_{h}}^{2}+\|v\|_{\partial \Omega_{h}}^{2}}_{i \lesssim\|v\|_{h}^{2}}+\underbrace{\left\|\left(I-P_{1, \Omega}\right) v\right\|_{\partial \Omega_{h}}^{2}}_{i i \lesssim\|v\|_{h_{2}}}
\end{aligned}
$$

which together with (37) proves (36). Here we estimated term $i$ using the stabilization (22),

$$
\left\|\nabla^{2} v\right\|_{\Omega}^{2} \lesssim\left\|\nabla^{2} v\right\|_{O_{h}}^{2} \lesssim\left\|\nabla^{2} v\right\|_{\Omega_{h}}^{2}+\|v\|_{s_{h}}^{2} \lesssim\|v\|_{h}^{2}
$$

Finally, to estimate $i i$ we recall the following technical estimate, which we prove in the "Appendix" of this paper see also the appendix of [9],

$$
\|w\|_{\partial \Omega_{h}}^{2} \lesssim\|w\|_{\partial \Omega}^{2}+\delta^{1 / 2}\|\nabla w\|_{U_{\delta}(\partial \Omega) \cap O_{h}}^{2} \quad w \in H^{1}\left(O_{h}\right)
$$


where $\delta \sim h^{4}$, bounds the distance between $\partial \Omega$ and $\partial \Omega_{h}$, see (10). Note that the finite element functions are defined on $O_{h}$, that contains both $\Omega$ and $\Omega_{h}$, and $V_{h} \subset H^{1}\left(O_{h}\right)$. Setting $w=\left(I-P_{1, h}\right) v$, using (40), the stabilization estimate (22), we obtain

$$
\begin{aligned}
\left\|\left(I-P_{1, h}\right) v\right\|_{\partial \Omega_{h}}^{2} & \lesssim\left\|\left(I-P_{1, h}\right) v\right\|_{\partial \Omega}^{2}+\delta^{1 / 2}\left\|\nabla\left(I-P_{1, h}\right) v\right\|_{U_{\delta}(\partial \Omega) \cap O_{h}}^{2} \\
& \lesssim\left\|\nabla^{2} v\right\|_{\Omega}^{2}+h^{2}\left\|\nabla\left(I-P_{1, h}\right) v\right\|_{O_{h}}^{2} \\
& \lesssim\left\|\nabla^{2} v\right\|_{\Omega}^{2}+h^{2}\left(\left\|\nabla\left(I-P_{1, h}\right) v\right\|_{\Omega}^{2}+\left\|\left(I-P_{1, h}\right) v\right\|_{s_{h}}^{2}\right) \\
& \lesssim\left\|\nabla^{2} v\right\|_{\Omega}^{2}+h^{2}\left(\left\|\nabla^{2} v\right\|_{\Omega}^{2}+\|v\|_{s_{h}}^{2}\right) \\
& \lesssim\left\|\nabla^{2} v\right\|_{\Omega_{h}}^{2}+h^{2}\|v\|_{s_{h}}^{2} \\
& \lesssim\|v\|_{h}^{2}
\end{aligned}
$$

where we finally used (44) to estimate $\left\|\nabla^{2} v\right\|_{\Omega}$ and the fact that $\left\|\left(I-P_{1, \Omega}\right) v\right\|_{s_{h}}=\|v\|_{s_{h}}$. This completes the verification.

\section{Interpolation}

Let $I_{h}: C^{1}\left(\mathbb{R}^{2}\right) \rightarrow V_{h}$ be the standard element wise interpolant associated with the degrees of freedom in $V_{h}$. Then we have the estimate

$$
\left\|v-I_{h} v\right\|_{H^{m}(T)} \lesssim h^{4-m}\|v\|_{H^{4}(T)} \quad m=0,1,2,3
$$

To construct an interpolation operator for cut elements we recall that given $v \in H^{s}(\Omega)$ there is an extension operator $E: H^{s}(\Omega) \rightarrow H^{s}\left(\mathbb{R}^{2}\right)$ such that

$$
\|E v\|_{H^{s}\left(\mathbb{R}^{2}\right)} \lesssim\|v\|_{H^{s}(\Omega)}
$$

for all $s>0$, cf. [21]. For simplicity we will often use the notation $w=E w$ for the extension of $w \in H^{s}(\Omega)$ to $\mathbb{R}^{2}$.

We define the interpolation operator

$$
C^{1}(\Omega) \ni v \mapsto I_{h}(E v)=\pi_{h} v \in V_{h}
$$

Combining (52) with (53) we obtain the interpolation error estimate

$$
\left\|v-\pi_{h} v\right\|_{H^{m}\left(\mathcal{T}_{h}\right)} \lesssim h^{4-m}\|v\|_{H^{4}(\Omega)} \quad m=0,1,2,3
$$

For the energy norm we have the estimate

$$
\left\|v-\pi_{h} v\right\|_{h} \lesssim h^{2}\|v\|_{H^{4}(\Omega)}
$$

\section{Verification of (56)}

Let $\eta=v-\pi_{h} v$ and recall that

$$
\|\eta\|_{h}^{2}=\|\eta\|_{\Omega_{h}}^{2}+\|\eta\|_{s_{h}}^{2}+h^{3}\|T(\eta)\|_{\partial \Omega_{h}}^{2}+h^{-3}\|\eta\|_{\partial \Omega_{h}}^{2}
$$

The first term is directly estimated using the interpolation error estimate (55),

$$
\|\eta\|_{\Omega_{h}}^{2} \lesssim h^{4}\|v\|_{H^{4}(\Omega)}^{2}
$$

For the second term we employ the trace inequality

$$
\|w\|_{\partial T}^{2} \lesssim h^{-1}\|w\|_{T}^{2}+h\|\nabla w\|_{T}^{2}
$$


to conclude that

$$
\begin{aligned}
\|\eta\|_{s_{h}}^{2} & =\sum_{j=2}^{3} h^{2 j-3}\left\|\left[\nabla_{n_{F}}^{j} \eta\right]\right\|_{\mathcal{F}_{h, B}}^{2} \\
& \lesssim \sum_{j=2}^{3} h^{2 j-3}\left(h^{-1}\left\|\nabla_{h_{F}}^{j} \eta\right\|_{\mathcal{T}_{h}\left(\mathcal{F}_{h, B}\right)}^{2}+h\left\|\nabla_{h_{F}}^{j+1} \eta\right\|_{\mathcal{T}_{h}\left(\mathcal{F}_{h, B}\right)}^{2}\right. \\
& \lesssim \sum_{j=2}^{3} h^{2 j-4}\left(\left\|\nabla^{j} \eta\right\|_{\mathcal{T}_{h}}^{2}+h^{2}\left\|\nabla^{j+1} \eta\right\|_{\mathcal{T}_{h}}^{2}\right) \\
& \lesssim \sum_{j=2}^{3} h^{2 j-4} h^{2(4-j)}\|v\|_{H^{4}(\Omega)}^{2} \lesssim h^{4}\|v\|_{H^{4}(\Omega)}^{2}
\end{aligned}
$$

For the third term we use the cut trace inequality (26) and the interpolation estimate (55),

$$
\begin{aligned}
h^{3}\|T(\eta)\|_{\partial \Omega_{h}}^{2} & \lesssim h^{3}\left(h^{-1}\left\|\nabla^{3} \eta\right\|_{\mathcal{T}_{h}\left(\partial \Omega_{h}\right)}^{2}+h\left\|\nabla^{4} \eta\right\|_{\mathcal{T}_{h}\left(\partial \Omega_{h}\right)}^{2}\right) \\
& \lesssim h^{2}\left\|\nabla^{3} \eta\right\|_{\mathcal{T}_{h}}^{2}+h^{4}\left\|\nabla^{4} \eta\right\|_{\mathcal{T}_{h}}^{2} \lesssim h^{4}\|v\|_{H^{4}(\Omega)}^{2}
\end{aligned}
$$

where $\mathcal{T}_{h}\left(\mathcal{F}_{h, B}\right) \subset \mathcal{T}_{h}$ is the set of elements with a face in $\mathcal{F}_{h, B}$. Finally, the fourth term is estimated in the same way as the third,

$$
\begin{aligned}
h^{-3}\|\eta\|_{\partial \Omega_{h}}^{2} & \lesssim h^{-3}\left(h^{-1}\|\eta\|_{\mathcal{T}_{h}\left(\partial \Omega_{h}\right)}^{2}+h\|\nabla \eta\|_{\mathcal{T}_{h}\left(\partial \Omega_{h}\right)}^{2}\right) \\
& \lesssim h^{-4}\|\eta\|_{\mathcal{T}_{h}}^{2}+h^{-2}\|\nabla \eta\|_{\mathcal{T}_{h}}^{2} \lesssim h^{4}\|v\|_{H^{4}(\Omega)}^{2}
\end{aligned}
$$

which completes the verification of (56).

\section{Consistency error estimate}

Lemma 1 Let $u$ be the exact solution to (1) with boundary conditions (5), and $u_{h}$ the finite element approximation defined by (13), then

$$
\left\|u-u_{h}\right\|_{h} \lesssim\left\|u-\pi_{h} u\right\|_{h}+\sup _{v \in V_{h} \backslash\{0\}} \frac{A_{h}\left(\pi_{h} u, v\right)-l_{h}(v)}{\|v\|_{h}}
$$

Proof Adding and subtracting an interpolant we obtain

$$
\left\|u-u_{h}\right\|_{h} \leq\left\|u-\pi_{h} u\right\|_{h}+\left\|\pi_{h} u-u_{h}\right\|_{h}
$$

Using coercivity we can estimate the second term on the right hand side as follows

$$
\begin{aligned}
\left\|\pi_{h} u-u_{h}\right\|_{h} & \leq \sup _{v \in V_{h} \backslash\{0\}} \frac{A_{h}\left(\pi_{h} u-u_{h}, v\right)}{\|v\|_{h}} \\
& \leq \sup _{v \in V_{h} \backslash\{0\}} \frac{A_{h}\left(\pi_{h} u-u, v\right)}{\|v\|_{h}}+\sup _{v \in V_{h} \backslash\{0\}} \frac{A_{h}\left(\pi_{h} u-u_{h}, v\right)}{\|v\|_{h}} \\
& \leq\left\|\pi_{h} u-u\right\|_{h}+\sup _{v \in V_{h} \backslash\{0\}} \frac{A_{h}\left(\pi_{h} u, v\right)-l_{h}(v)}{\|v\|_{h}}
\end{aligned}
$$

where we added and subtracted $u$ in the numerator and for the first term used the estimate $A_{h}\left(\pi_{h} u-u, v\right) \lesssim\left\|\pi_{h} u-u\right\|_{h}\|v\|_{h}$ and for the second used (13) to eliminate $u_{h}$. Combining the estimates the desired result follows directly. 
Lemma 2 Let $\varphi \in H^{4}\left(\mathbb{R}^{2}\right)$ and $v \in V+V_{h}$, then

$$
\begin{aligned}
(\nabla \cdot(M(\varphi) \cdot \nabla), v)_{\Omega_{h}}= & (M(\varphi), \epsilon(\nabla v))_{\Omega_{h}}-\left(M_{n n}(\varphi), \nabla_{n} v\right)_{\partial \Omega_{h}} \\
& +(T(\varphi), v)_{\partial \Omega_{h}}+\left(\left[M_{n t}\right], v\right)_{\mathcal{X}_{h}}
\end{aligned}
$$

where, for $x \in \mathcal{X}_{h}$ (the set of points where $\partial \Omega_{h}$ is not smooth), $\left[M_{n t}\right]_{x}$ is defined by

$$
\left.\left[M_{n t}\right]\right|_{x}=M(x)_{n_{h}^{+}} t_{h}^{+}-M(x)_{n_{h}^{-}} t_{h}^{-}
$$

with $n_{h}^{ \pm}$and $t_{h}^{ \pm}$the left and right limits to tangent and normal to the discrete boundary $\partial \Omega_{h}$ at $x \in \mathcal{X}_{h}$. In the case of $C^{1}$ boundary $\left(v,\left[M_{n t}\right]\right)_{\mathcal{X}_{h}}=0$.

Proof Using the simplified notation $M=M(\varphi)$ and $T=T(\varphi)$ for brevity we obtain by integrating by parts

$$
\begin{aligned}
(\nabla \cdot(M \cdot \nabla), v)_{\Omega_{h}} & =\left((M \cdot \nabla)_{n}, v\right)_{\partial \Omega_{h}}-(M \cdot \nabla, \nabla v)_{\Omega_{h}} \\
& =\left((M \cdot \nabla)_{n}, v\right)_{\partial \Omega_{h}}-\left(M_{n}, \nabla v\right)_{\partial \Omega_{h}}+(M, \epsilon(\nabla v))_{\Omega_{h}}
\end{aligned}
$$

Splitting $\nabla v$ in tangent and normal contributions on $\partial \Omega_{h}$, we have the identity

$$
\begin{aligned}
\left(\nabla v, M_{n}\right)_{\partial \Omega_{h} \cap T} & =\left(\nabla_{n} v, M_{n n}\right)_{\partial \Omega_{h} \cap T}+\left(\nabla_{t} v, M_{n t}\right)_{\partial \Omega_{h} \cap T} \\
& =\left(\nabla_{n} v, M_{n n}\right)_{\partial \Omega_{h} \cap T}-\left(v, \nabla_{t} M_{n t}\right)_{\partial \Omega_{h} \cap T}+\left(v, M_{n t} t \cdot v\right)_{\partial\left(\partial \Omega_{h} \cap T\right)}
\end{aligned}
$$

where we integrated by parts along the curve segments $\partial \Omega_{h} \cap T$, and $v$ is the exterior unit tangent vector to $\partial \Omega_{h} \cap T$. Summing over all elements that intersect $\partial \Omega_{h}$, we obtain the identity

$$
\left(\nabla v, M_{n}\right)_{\partial \Omega_{h}}=\left(\nabla_{n} v, M_{n n}\right)_{\partial \Omega_{h}}-\left(v, \nabla_{t} M_{n t}\right)_{\partial \Omega_{h}}+\left(v,\left[M_{n t}\right]\right)_{\mathcal{X}_{h}}
$$

Combining (75) and (78), we obtain

$$
\begin{aligned}
(v, \nabla \cdot(M \cdot \nabla))_{\Omega_{h}}= & (\epsilon(\nabla v), M)_{\Omega_{h}}-\left(\nabla_{n} v, M_{n n}\right)_{\partial \Omega_{h}} \\
& +\left(v,(M \cdot \nabla)_{n}+\nabla_{t} M_{n t}\right)_{\partial \Omega_{h}}-\left(v,\left[M_{n t}\right]\right)_{\mathcal{X}_{h}}
\end{aligned}
$$

and setting $T=(M \cdot \nabla)_{n}+\nabla_{t} M_{n t}$ we obtain the desired result.

Lemma 3 Let $u$ be the exact solution to (1) with boundary conditions (5), then there is a constant such that for all $v \in V_{h}$,

$$
A_{h}(u, v)-l_{h}(v) \lesssim h^{4}\|u\|_{H^{4}(\Omega)}\|v\|_{h, \star} \lesssim h^{5 / 2}\|u\|_{H^{4}(\Omega)}\|v\|_{h}
$$

where $\|v\|_{h, \star}$ is the norm

$$
\|v\|_{h, \star}^{2}=\|v\|_{h}^{2}+\|T(v)\|_{\partial \Omega_{h}}^{2}+h^{-6}\|v\|_{\partial \Omega_{h}}^{2} \leq\left(1+h^{-3}\right)\|v\|_{h}^{2}
$$


Proof Using the definition (14), the fact that $s_{h}(u, v)=0$ for $u \in H^{4}(\Omega)$, and the partial integration identity (71) we obtain

$$
\begin{aligned}
A_{h}(u, v)-l_{h}(v)= & (M(u), \epsilon(\nabla v))_{\Omega_{h}}+(T(u), v)_{\partial \Omega_{h}}+(u, T(v))_{\partial \Omega_{h}} \\
& +\gamma h^{-3}(u, v)_{\partial \Omega_{h}}-(\nabla \cdot(M(u) \cdot \nabla), v)_{\Omega_{h}} \\
= & \left(M_{n n}(u), \nabla_{h} v\right)_{\partial \Omega_{h}}+\left(\left[M_{n t}\right], v\right)_{\mathcal{X}_{h}} \\
& +(u, T(v))_{\partial \Omega_{h}}+\gamma h^{-3}(u, v)_{\partial \Omega_{h}} \\
= & I+I I+I I I+I V
\end{aligned}
$$

Before turning to the estimates of $I-I V$ we first note that for $w \in H_{0}^{1}(\Omega)$, with extension to $\mathbb{R}^{2}$ also denoted by $w$, we may apply (45) and the stability (53) of the extension that

$$
\|w\|_{\partial \Omega_{h}}^{2} \lesssim \delta\|w\|_{H^{1}\left(U_{\delta}(\partial \Omega)\right)}^{2} \lesssim \delta\|w\|_{H^{1}\left(U_{\delta_{0}}(\partial \Omega) \cup \Omega\right)}^{2} \lesssim \delta\|w\|_{H^{1}(\Omega)}^{2}
$$

with $\delta \sim h^{4}$. Note that here we do not need to restrict the norms to $O_{h}$ as in (45) since the extended function is defined on $\mathbb{R}^{2}$. See $[9$, Appendix] for detailed derivations. Furthermore, for more regular functions such that $w \in H^{2}(\Omega)$ with $w=0$ on $\partial \Omega$, we may strengthen the estimate as follows

$$
\|w\|_{\partial \Omega_{h}}^{2} \lesssim \delta\|\nabla w\|_{L^{2}\left(U_{\delta}(\partial \Omega)\right)}^{2} \lesssim \delta^{2} \sup _{t \in(-\delta, \delta)}\|\nabla w\|_{\partial \Omega_{t}}^{2} \lesssim \delta^{2}\|w\|_{H^{2}(\Omega)}
$$

where $\partial \Omega_{t}=\left\{x \in \mathbb{R}^{2} \mid \rho(x)=t\right\}$ for $t \in\left(-\delta_{0}, \delta_{0}\right)$, are level sets of $\rho$, and again $\delta \sim h^{4}$. In the last step we used a version of (45) to conclude that

$$
\begin{aligned}
& \|\nabla w\|_{\partial \Omega_{t}}^{2} \lesssim\|\nabla w\|_{\partial \Omega}^{2}+t\left\|\nabla^{2} w\right\|_{U_{t}(\partial \Omega)}^{2} \\
& \quad \lesssim\|w\|_{H^{2}(\Omega)}^{2}+\delta_{0}\left\|\nabla^{2} w\right\|_{U_{\delta_{0}}(\partial \Omega)}^{2} \lesssim\|w\|_{H^{2}(\Omega)}^{2}
\end{aligned}
$$

where we used a trace inequality on $\Omega$ and the stability (53) of the extension operator.

I. Using Cauchy-Schwarz followed by (89) with $w=M_{n n}(u)$, we get

$$
\left(M_{n n}(u), \nabla_{n} v\right)_{\partial \Omega_{h}} \lesssim\left\|M_{n n}(u)\right\|_{\partial \Omega_{h}}\left\|\nabla_{n} v\right\|_{\partial \Omega_{h}} \lesssim \delta\|u\|_{H^{4}(\Omega)}\|v\|_{h}
$$

Here we used the estimate

$$
\left\|\nabla_{h} v\right\|_{\partial \Omega_{h}} \lesssim\|v\|_{h}
$$

which we derive by applying (45) with $w=\nabla v$,

$$
\begin{aligned}
\left\|\nabla_{h} v\right\|_{\partial \Omega_{h}}^{2} & \lesssim\|\nabla v\|_{\partial \Omega_{h}}^{2} \lesssim\|\nabla v\|_{\partial \Omega}^{2}+\delta\left\|\nabla^{2} v\right\|_{U_{\delta}(\partial \Omega) \cap O_{h}}^{2} \\
& \lesssim\|v\|_{H^{2}(\Omega)}^{2}+\delta\left\|\nabla^{2} v\right\|_{U_{\delta}(\partial \Omega) \cap O_{h}}^{2} \lesssim\|v\|_{O_{h}}^{2} \lesssim\|v\|_{h}^{2}
\end{aligned}
$$

where we used the trace inequality $\|\nabla v\|_{\partial \Omega} \lesssim\|\nabla v\|_{H^{1}(\Omega)} \lesssim\|v\|_{H^{2}(\Omega)}$, and at last the Poincaré inequality (36). 
II. Using the assumption on the accuracy of the discrete normal (11) we have for each $x \in \mathcal{X}_{h}$,

$$
\left|\left[M_{n t}\right]\right|=M_{n_{h} t_{h}}^{+}-M_{n_{h} t_{h}}^{-}=M_{n_{h} t_{h}}^{+}-M_{n t}+M_{n t}-M_{n_{h} t_{h}}^{-}
$$

where the first term on the right hand side can be estimated as follows

$$
\left|M_{n_{h} t_{h}}^{+}-M_{n t}\right| \leq\left|\left(n_{h}-n\right) \cdot M \cdot t_{h}\right|+\left|n \cdot M \cdot\left(t_{h}-t\right)\right| \lesssim h^{3}|M|
$$

We then have

$$
\begin{aligned}
\left(\left[M_{n t}\right], v\right)_{\mathcal{X}_{h}} & \leq\left\|\left[M_{n t}\right]\right\|_{\mathcal{X}_{h}}\|v\|_{\mathcal{X}_{h}} \lesssim h^{3}\|M\|_{\mathcal{X}_{h}}\|v\|_{\mathcal{X}_{h}} \\
& \lesssim h^{2} h^{1 / 2}\|M\|_{\mathcal{X}_{h}} h^{1 / 2}\|v\| \mathcal{X}_{h} \\
& \lesssim h^{2}\|M\|_{L^{\infty}\left(\mathcal{X}_{h}\right)} h^{2}\|v\|_{h} \lesssim h^{4}\|u\|_{H^{4}(\Omega)}\|v\|_{h}
\end{aligned}
$$

where we used the fact that the number of elements, denoted by $\left|\mathcal{X}_{h}\right|$, in $\mathcal{X}_{h}$ satisfies $\left|\mathcal{X}_{h}\right| \sim h^{-1}$, and the Sobolev inequality [13] followed by the stability (53) of the extension operator to obtain

$$
h\|M\|_{L^{\infty}\left(\mathcal{X}_{h}\right)}^{2} \lesssim\|u\|_{W_{\infty}^{2}\left(\mathbb{R}^{2}\right)}^{2} \lesssim\|u\|_{H^{3+\epsilon}\left(\mathbb{R}^{2}\right)}^{2} \lesssim\|u\|_{H^{4}\left(\mathbb{R}^{2}\right)}^{2} \lesssim\|u\|_{H^{4}(\Omega)}^{2}
$$

and the estimate

$$
h\|v\|_{\mathcal{X}_{h}}^{2} \lesssim h^{3}\|v\|_{h}^{2} \quad v \in V_{h}
$$

To verify (100) we shall employ an inverse inequality locally using a linear approximation of the boundary. To that end consider $x \in \mathcal{X}_{h}$ and let $B_{r}(x)$ be a ball of radius $r \sim h$ centred at $x$. Let $T_{x} \in \mathcal{T}_{h}$ be one of the elements such that $x \in \partial T_{x} \cap \partial \Omega_{h}$ and given $v \in V_{h}$ let $v_{x} \in Q_{3}\left(\mathbb{R}^{2}\right)$ be the extension to $\mathbb{R}^{2}$ of $\left.v\right|_{T_{x}} \in Q_{3}\left(T_{x}\right)$ such that $\left.v_{x}\right|_{T_{x}}=\left.v\right|_{T_{x}}$. Let $\Gamma_{x}$ be a line through $x$ such that $\|\rho\|_{L^{\infty}\left(\Gamma_{x} \cap B_{r}(x)\right)} \lesssim h^{2}$. Such a line exists since $\partial \Omega$ is smooth and linear approximation is of second order locally. We then have

$$
h\|v\|_{\mathcal{X}_{h}}^{2} \lesssim \sum_{x \in \mathcal{X}_{h}} h|v(x)|^{2} \lesssim \sum_{x \in \mathcal{X}_{h}}\left\|v_{x}\right\|_{\Gamma_{x} \cap B_{r}(x)}^{2}
$$

where we used the fact that $\left|\Gamma_{x} \cap B_{r}(x)\right| \sim h$. In order to employ a local version of (45) we define the cylindrical tubular neighborhood

$$
U_{\delta, x}=U_{\delta_{1}}\left(p\left(\Gamma_{x} \cap B_{r}(x)\right)\right) \quad \delta \in\left(0, \delta_{0}\right]
$$

over $p\left(\Gamma_{x} \cap B_{r}(x)\right) \subset \partial \Omega$, where $p: U_{\delta_{0}}(\partial \Omega) \rightarrow \partial \Omega$ is the closest point mapping. Then there is $\delta_{1} \sim h^{2}$ such that $\Gamma_{x} \cap B_{r}(x) \subset U_{\delta_{1}, x}$ and $\partial \Omega_{h} \cap U_{\delta_{0}, x} \subset U_{\delta_{1}, x}$, since $\Gamma_{x}$ is $O\left(h^{2}\right)$ accurate locally and $\partial \Omega_{h}$ is $O\left(h^{4}\right)$ accurate. We obtain

$$
\begin{aligned}
\left\|v_{x}\right\|_{\Gamma_{x} \cap B_{r}(x)}^{2} & \lesssim\left\|v_{x}\right\|_{\partial \Omega_{h} \cap U_{\delta_{1}, x}}^{2}+\delta_{1}\left\|\nabla v_{x}\right\|_{U_{\delta_{1}, x}}^{2} \\
& \lesssim\left\|v_{x}\right\|_{\partial \Omega_{h} \cap U_{\delta_{1}, x}}^{2}+\delta_{1}\left\|\nabla v_{x}\right\|_{T_{x}}^{2} \\
& \lesssim\|v\|_{\partial \Omega_{h} \cap U_{\delta_{1}, x}}^{2}+\left\|v_{x}-v\right\|_{\partial \Omega_{h} \cap B_{r}(x)}^{2}+\delta_{1}\|\nabla v\|_{T_{x}}^{2}
\end{aligned}
$$


then we added and subtracted $v$ in the first term, used the triangle inequality, the inverse estimate $\left\|\nabla v_{x}\right\|_{U_{\delta_{1}, x}}^{2} \lesssim\left\|\nabla v_{x}\right\|_{T_{x}}$ which holds since $v_{x}$ is a polynomial, and $U_{\delta_{1}, x} \subset B_{r^{\prime}}(x)$ for some ball of radius $r^{\prime} \sim h$, and finally we noted that $v_{x}=v$ on $T_{x}$. Inequality (103) is an application of (154). Inserting (105) in (101) we get

$$
\begin{aligned}
h\|v\|_{\mathcal{X}_{h}}^{2} & \lesssim \sum_{x \in \mathcal{X}_{h}}\|v\|_{\partial \Omega_{h} \cap U_{\delta_{1}, x}}^{2}+\left\|v_{x}-v\right\|_{\partial \Omega_{h} \cap U_{\delta_{1}, x}}^{2}+\delta_{1}\|\nabla v\|_{T_{x}}^{2} \\
& \lesssim\|v\|_{\partial \Omega_{h}}^{2}+\underbrace{\sum_{x \in \mathcal{X}_{h}}\left\|v_{x}-v\right\|_{\partial \Omega_{h} \cap B_{r}(x)}^{2}}_{i \lesssim h^{4}\|v\|_{s_{h}}^{2}}+\underbrace{\sum_{x \in \mathcal{X}_{h}} \delta_{1}\|\nabla v\|_{T_{x}}^{2}}_{i i h^{3}\|v\|_{H^{2}(\Omega)}^{2}+h^{4}\|v\|_{s_{h}}^{2}} \\
& \lesssim\|v\|_{\partial \Omega_{h}}^{2}+h^{3}\|v\|_{H^{2}(\Omega)}^{2}+h^{4}\|v\|_{s_{h}}^{2} \\
& \lesssim h^{3}\|v\|_{h}^{2}
\end{aligned}
$$

which establishes (100). Here we used the fact that the number of cylinders $U_{\delta_{1}, y}, y \in$ $\mathcal{X}_{h}$, that intersect $U_{\delta_{1}, x}$ is uniformly bounded independent of $x \in \mathcal{X}_{h}$ and $h \in\left(0, h_{0}\right]$. We also used certain estimates of terms $i$ and $i i$, which we verify next.

i. Let $\mathcal{T}_{h, x}=\mathcal{T}_{h}\left(\partial \Omega_{h} \cap B_{r}(x)\right), \mathcal{F}_{h, x}$ be the interior faces in $\mathcal{T}_{h, x}$, and $s_{h, x}$ be defined by (16) with $\mathcal{F}_{h, B}$ replaced by $\mathcal{F}_{h, x}$. Using the estimate

$$
\left\|v_{x}-v\right\|_{\mathcal{T}_{h, x}}^{2} \lesssim h^{4}\|v\|_{s_{h, x}}^{2}
$$

which is a local version of (22) on the patch $\mathcal{T}_{h, x}$, we get

$$
i \lesssim \sum_{x \in \mathcal{X}_{h}}\left\|v_{x}-v\right\|_{\mathcal{T}_{h, x}}^{2} \lesssim \sum_{x \in \mathcal{X}_{h}} h^{4}\|v\|_{s_{h, x}}^{2} \lesssim h^{4}\|v\|_{s_{h}}^{2}
$$

ii. We first note that

$$
\begin{aligned}
i i=\sum_{x \in \mathcal{X}_{h}} \delta_{1}\|\nabla v\|_{T_{x}}^{2} & \lesssim \delta_{1}\|\nabla v\|_{\mathcal{T}_{h}\left(\partial \Omega_{h}\right)}^{2} \\
& \lesssim \delta_{1}\left(\|\nabla v\|_{\mathcal{N}_{h}\left(\mathcal{T}_{h}\left(\partial \Omega_{h}\right)\right) \backslash \mathcal{T}_{h}\left(\partial \Omega_{h}\right)}^{2}+h^{2}\|v\|_{S_{h}}^{2}\right)
\end{aligned}
$$

where $\mathcal{N}_{h}\left(\mathcal{T}_{h}\left(\partial \Omega_{h}\right)\right)$ is the set of elements that share a node with an element in $\mathcal{T}_{h}\left(\partial \Omega_{h}\right)$, and we used the stabilization. We can then choose $\delta_{2} \sim h$ such that

$$
\mathcal{N}_{h}\left(\mathcal{T}_{h}\left(\partial \Omega_{h}\right)\right) \backslash \mathcal{T}_{h}\left(\partial \Omega_{h}\right) \subset U_{\delta_{2}}(\partial \Omega) \cap \Omega
$$

We now proceed in a similar way as in (89),

$$
\begin{aligned}
\|\nabla v\|_{\mathcal{N}_{h}\left(\mathcal{T}_{h}\left(\partial \Omega_{h}\right)\right) \backslash \mathcal{T}_{h}\left(\partial \Omega_{h}\right)}^{2} & \lesssim\|\nabla v\|_{U_{\delta_{2}}(\partial \Omega) \cap \Omega}^{2} \\
& \lesssim \sup _{t \in\left(0,-\delta_{2}\right)} \delta_{2}\|\nabla v\|_{\partial \Omega_{t}}^{2} \lesssim \delta_{2}\|v\|_{H^{2}(\Omega)}^{2}
\end{aligned}
$$

where we used the estimate (90) in the last step. Inserting (114) in (112) and using that $\delta_{1} \sim h^{2}$ and $\delta_{2} \sim h$, we obtain

$$
i i \lesssim \delta_{1}\left(\delta_{2}\|v\|_{H^{2}(\Omega)}^{2}+h^{2}\|v\|_{s_{h}}^{2}\right) \lesssim h^{3}\|v\|_{H^{2}(\Omega)}^{2}+h^{4}\|v\|_{s_{h}}^{2}
$$


III. Using (89) with $w=u$ and recalling $\delta \sim h^{4}$,

$$
\begin{aligned}
(u, T(v))_{\partial \Omega_{h}} & \leq\|u\|_{\partial \Omega_{h}}\|T(v)\|_{\partial \Omega_{h}} \lesssim \delta\|u\|_{H^{4}(\Omega)}\|T(v)\|_{\partial \Omega_{h}} \\
& \lesssim \delta h^{-3 / 2}\|u\|_{H^{4}(\Omega)} h^{3 / 2}\|T(v)\|_{\partial \Omega_{h}} \lesssim h^{5 / 2}\|u\|_{H^{4}(\Omega)}\|v\|_{h}
\end{aligned}
$$

IV. Proceeding in the same way as for Term III,

$$
\begin{aligned}
h^{-3}(u, v)_{\partial \Omega_{h}} & \lesssim h^{-3}\|u\|_{\partial \Omega_{h}}\|v\|_{\partial \Omega_{h}} \lesssim \delta h^{-3}\|u\|_{H^{4}(\Omega)}\|v\|_{\partial \Omega_{h}} \\
& \lesssim \delta h^{-3 / 2}\|u\|_{H^{4}(\Omega)}\|v\|_{h} \lesssim h^{5 / 2}\|u\|_{H^{4}(\Omega)}\|v\|_{h}
\end{aligned}
$$

Combining the estimates we find that

$$
\begin{aligned}
A_{h}(u, v)-l_{h}(v) & \lesssim h^{4}\|u\|_{H^{4}(\Omega)}\|v\|_{h}+h^{5 / 2}\|u\|_{H^{4}(\Omega)}\|v\|_{h} \\
& \lesssim h^{5 / 2}\|u\|_{H^{4}(\Omega)}\|v\|_{h}
\end{aligned}
$$

which completes the proof.

\section{Error estimates}

Theorem 1 The finite element solution defined by (13) satisfies

$$
\left\|u-u_{h}\right\|_{h} \lesssim h^{2}\|u\|_{H^{4}(\Omega)}
$$

Proof Using the second bound of (81) in (66) followed by the interpolation error bound (56) we directly get the desired estimate.

Theorem 2 The finite element solution defined by (13) satisfies

$$
\left\|u-u_{h}\right\|_{\Omega_{h}} \lesssim h^{4}\|u\|_{H^{4}(\Omega)}
$$

Proof Adding and subtracting an interpolant and using the interpolation error estimate (55) we have the estimate

$$
\begin{aligned}
\left\|u-u_{h}\right\|_{\Omega_{h}} & \leq\left\|u-\pi_{h} u\right\|_{\Omega_{h}}+\left\|\pi_{h} u-u_{h}\right\|_{\Omega_{h}} \\
& \lesssim h^{4}\|u\|_{H^{4}(\Omega)}+\left\|\pi_{h} u-u_{h}\right\|_{\Omega_{h}}
\end{aligned}
$$

In order to estimate $\left\|\pi_{h} u-u_{h}\right\|_{\Omega_{h}}$ we let $\phi_{h} \in V_{h}$ be the solution to the discrete dual problem

$$
(v, \psi)_{\Omega_{h}}=A_{h}\left(v, \phi_{h}\right) \quad \forall v \in V_{h}
$$

Setting $v=\pi_{h} u-u_{h}$ we obtain the error representation

$$
\begin{aligned}
\left(\pi_{h} u-u_{h}, \psi\right)_{\Omega_{h}}= & A_{h}\left(\pi_{h} u-u_{h}, \phi_{h}\right) \\
= & A_{h}\left(\pi_{h} u-u, \phi_{h}\right)+A_{h}\left(u-u_{h}, \phi_{h}\right) \\
= & \underbrace{A_{h}\left(\pi_{h} u-u, \phi_{h}-\phi\right)}_{I}+\underbrace{A_{h}\left(\pi_{h} u-u, \phi\right)}_{I I} \\
& +\underbrace{A_{h}\left(u, \phi_{h}\right)-l_{h}\left(\phi_{h}\right)}_{I I I}
\end{aligned}
$$


Here $\phi$ is the solution to the continuous dual problem

$$
\nabla \cdot(\sigma(\nabla \phi) \cdot \nabla)=\psi \quad \text { in } \Omega, \quad \phi=M_{n n}(\phi)=0 \quad \text { on } \partial \Omega
$$

extended to $\mathbb{R}^{2}$ using the stable extension operator, see (53).

I. Since $\phi_{h}$ is the finite element approximation of $\phi$ we have the error estimate

$$
\left\|\phi-\phi_{h}\right\|_{h} \lesssim h^{2}\|\phi\|_{H^{4}(\Omega)} \lesssim h^{2}\|\psi\|_{\Omega}
$$

where we used elliptic regularity (9), which directly gives

$$
A_{h}\left(\pi_{h} u-u, \phi_{h}-\phi\right) \leq\left\|\pi_{h} u-u\right\|_{h}\left\|\phi_{h}-\phi\right\|_{h} \lesssim h^{4}\|u\|_{H^{4}(\Omega)}\|\psi\|_{\Omega}
$$

II. Using the fact that $s_{h}\left(\pi_{h} u-u, \phi\right)=0$ since $\phi \in H^{4}(\Omega)$, the partial integration formula (71), the Cauchy-Schwarz inequality, and the interpolation error estimates we obtain

$$
\begin{aligned}
& A_{h}\left(\pi_{h} u-u, \phi\right)=\left(\pi_{h} u-u, \psi\right)_{\Omega_{h}}-\left(\nabla_{n}\left(\pi_{h} u-u\right), M_{n n}(\phi)\right)_{\partial \Omega_{h}} \\
& +\left(T\left(\pi_{h} u-u\right), \phi\right)_{\partial \Omega_{h}}+\gamma h^{-3}\left(\pi_{h} u-u, \phi\right)_{\partial \Omega_{h}} \\
& \leq\left\|\pi_{h} u-u\right\|_{\Omega_{h}}\|\psi\|_{\Omega_{h}}+\left\|\nabla_{h}\left(\pi_{h} u-u\right)\right\|_{\partial \Omega_{h}}\left\|M_{n n}(\phi)\right\|_{\partial \Omega_{h}} \\
& +\left\|T\left(\pi_{h} u-u\right)\right\|_{\partial \Omega_{h}}\|\phi\|_{\partial \Omega_{h}}+\gamma h^{-3}\left\|\pi_{h} u-u\right\|_{\partial \Omega_{h}}\|\phi\|_{\partial \Omega_{h}} \\
& \lesssim h^{4}\|u\|_{H^{4}\left(\Omega_{h}\right)}\|\psi\|_{\Omega_{h}}+h^{5 / 2}\|u\|_{H^{4}\left(\Omega_{h}\right)}\left\|M_{n n}(\phi)\right\|_{\partial \Omega_{h}} \\
& +h^{1 / 2}\|u\|_{H^{4}\left(\Omega_{h}\right)}\|\phi\|_{\partial \Omega_{h}}+\gamma h^{-3} h^{7 / 2}\|u\|_{H^{4}\left(\Omega_{h}\right)}\|\phi\|_{\partial \Omega_{h}} \\
& \lesssim \underbrace{\left(h^{4}+h^{5 / 2} h^{2}+h^{1 / 2} h^{4}\right)}_{\lesssim h^{4}}\|u\|_{H^{4}\left(\Omega_{h}\right)}\|\psi\|_{\Omega_{h}}
\end{aligned}
$$

Here we used (88) with $\delta \sim h^{4}$ followed by the elliptic regularity (9) to conclude that

$$
\left\|M_{n n}(\phi)\right\|_{\partial \Omega_{h}} \lesssim \delta^{1 / 2}\|\phi\|_{H^{3}\left(U_{\delta}(\partial \Omega)\right)} \lesssim h^{2}\|\phi\|_{H^{4}(\Omega)} \lesssim h^{2}\|\psi\|_{\Omega}
$$

and using (89) we obtain

$$
\|\phi\|_{\partial \Omega_{h}} \lesssim \delta\|\phi\|_{H^{4}(\Omega)} \lesssim h^{4}\|\psi\|_{\Omega_{h}}
$$

III. Using (81) we obtain

$$
\left|A_{h}\left(u, \phi_{h}\right)-\left(f, \phi_{h}\right)_{\Omega_{h}}\right| \lesssim h^{4}\|u\|_{H^{4}(\Omega)}\left\|\phi_{h}\right\|_{h, \star} \lesssim h^{4}\|u\|_{H^{4}(\Omega)}\|\psi\|_{\Omega_{h}}
$$

We used the estimate

$$
\begin{aligned}
\left\|\phi_{h}\right\|_{h, \star}^{2} & \lesssim\left\|\phi_{h}-\phi\right\|_{h, \star}^{2}+\|\phi\|_{h, \star}^{2} \\
& \lesssim\left(1+h^{-3}\right)\left\|\phi_{h}-\phi\right\|_{h}^{2}+\|\phi\|_{h}^{2}+\|T(\phi)\|_{\partial \Omega_{h}}^{2}+h^{-6}\|\phi\|_{\partial \Omega_{h}}^{2} \\
& \lesssim\left(1+h^{-3}\right) h^{4}\|\phi\|_{H^{4}(\Omega)}^{2}+\|\phi\|_{h}^{2}+\left(1+h^{-6} \delta^{2}\right)\|\phi\|_{H^{4}(\Omega)}^{2} \\
& \lesssim\|\psi\|_{\Omega_{h}}^{2}
\end{aligned}
$$

where we used (89) to conclude that $\|T(\phi)\|_{\partial \Omega_{h}}^{2}+h^{-6}\|\phi\|_{\partial \Omega_{h}}^{2} \lesssim\left(1+h^{-6} \delta^{2}\right)\|\phi\|_{H^{4}(\Omega)}^{2}$. Collecting the estimates of Terms $I-I I I$ completes the proof. 


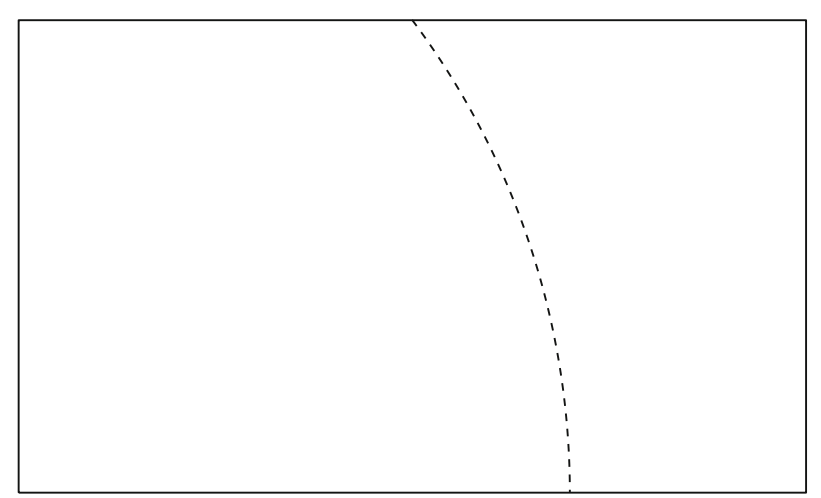

Fig. 2 Element intersected by the boundary (dashed)

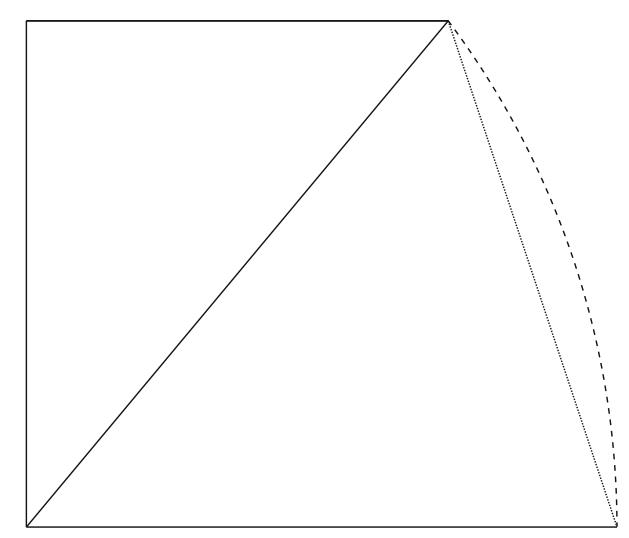

Fig. 3 Straight line approximation of the boundary (dotted) and triangulation for integration purposes

\section{Numerics}

\section{Implementation}

We consider two higher order approximations of the boundary: a piecewise cubic $C^{0}$ approximation or a piecewise cubic $C^{1}$ approximation. The steps to create the approximate boundary are as follows.

1. The elements cut by the boundary are located, Fig. 2 .

2. Straight segments connecting the intersection points between the boundary and the elements are established, and the geometrical object inside the domain is triangulated for ease if integration, Fig. 3.

3. The end points of the boundary segments and the inclinations at the endpoints (computed by use of tangent vectors) is used to obtain a $C^{1}$ interpolant of the boundary, Fig. 4. (This step is skipped in the case of a $C^{0}$ approximation of the boundary.)

4. The geometry is approximated by a cubic triangle, interpolating the exact boundary ( $C^{0}$ case) or the spline boundary $\left(C^{1}\right.$ case), Fig 5 .

Note that the approximation of the boundary may partly land outside the element. In such cases, the basis functions of the element containing the straight segment is used also outside of the element. 


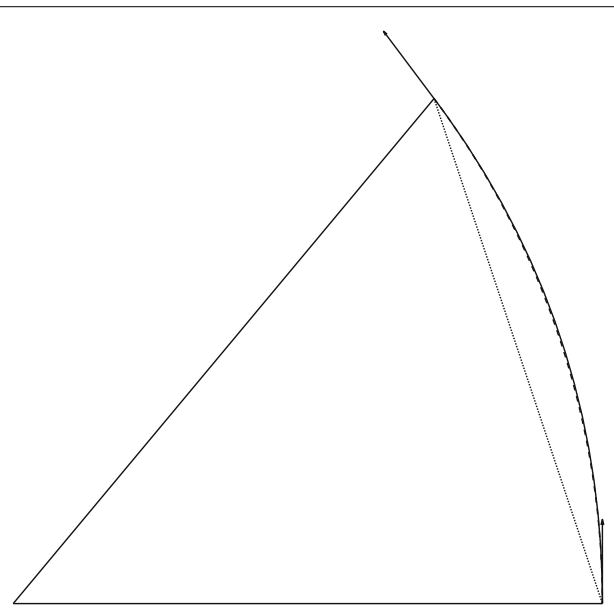

Fig. 4 Cubic spline approximation of the boundary (solid line)

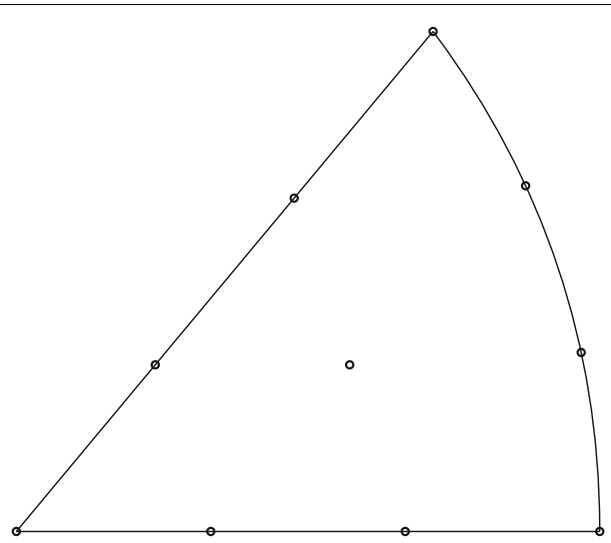

Fig. 5 Isoparametric cubic triangle approximation of the geometry

\section{Example}

We consider a circular simply supported plate under uniform load $p$. The plate is of radius $R=1 / 2$ and has its center at $x=1 / 2, y=1 / 2$. Defining $r$ as the distance from the midpoint we then have the exact solution

$$
u=\frac{p R^{4}}{64 \kappa}\left(1-\left(\frac{r}{R}\right)^{2}\right)\left(\frac{5+v}{1+v}-\left(\frac{r}{R}\right)^{2}\right)
$$

see, e.g., [18]. The constitutive parameters were chosen as $E=10^{2}, v=0.3, t=10^{-1}$, and the stabilization parameters as $\beta=10^{-2}, \gamma=10^{2}\left(2 \kappa+2 \kappa \nu(1-\nu)^{-1}\right)$.

We compare the convergence in normalized $\left(\left\|u-u_{h}\right\| /\|u\|\right) L_{2}, H^{1}$ and piecewise $H^{2}$ norms in Fig. 6. These norms are computed on the discrete geometry, for simplicity the straight segment geometry. The solid lines indicate second, third, and fourth order convergence, respectively from top to bottom, and we note that we observe a slightly higher than optimal rate of convercence of about $O\left(h^{1 / 2}\right)$ in all norms. We note that the continuity of the approximation of the boundary seems not to be crucial as the convergence curves are very close. In Fig. 7 we show an elevation of the solution on one of the meshes in the sequence. 


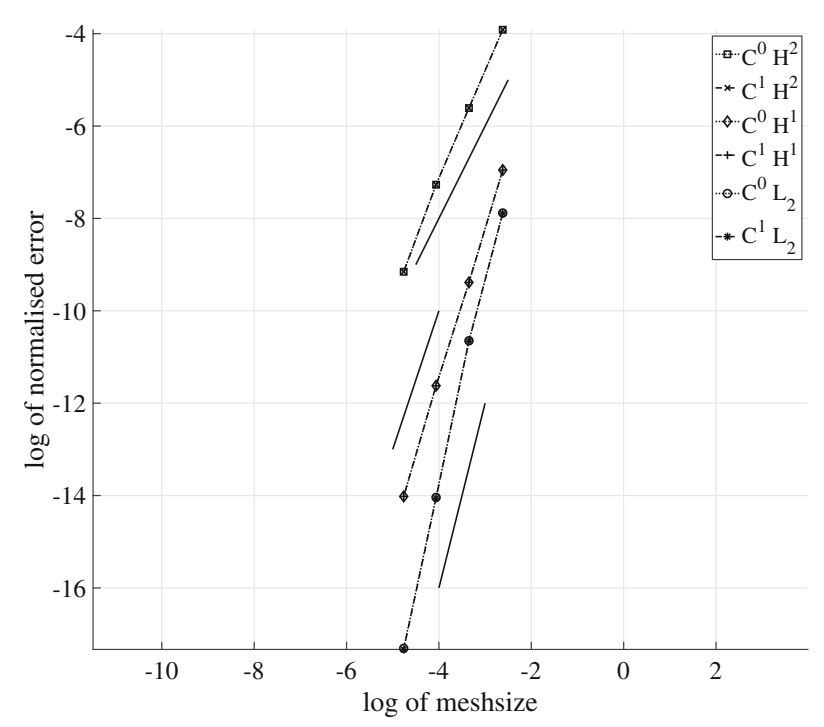

Fig. 6 Convergence in normalized $L_{2}, H^{1}$, and piecewise $H^{2}$ norms

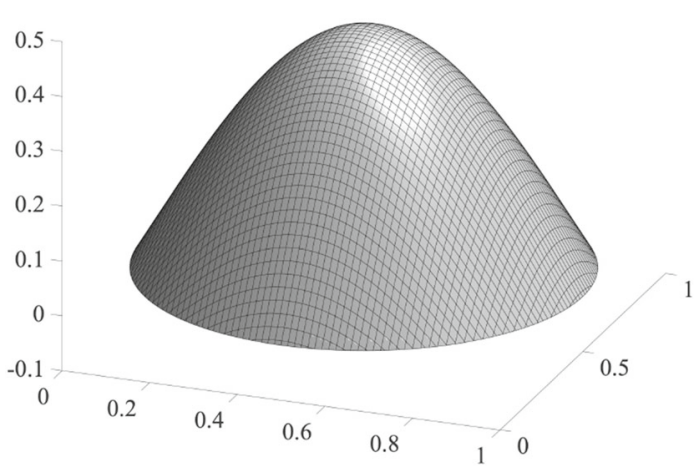

Fig. 7 Elevation of the discrete solution on one of the meshes in the sequence

We also show the influence of the parameter $\beta$ on a fixed mesh (coarse, 386 active nodes). In Fig. 8 we show the condition number as $\beta$ increases from its critical number, the number for which the system matrix is singular. For lower values of $\beta$ there are negative eigenvalues in the system matrix. We note that as $\beta$ increases, the condition number will eventually increase again after an initial drop. In Fig. 9 we show the $H^{1}$ error which is more sensitive to the increase in $\beta$. We remark that this effect, however, does not affect the convergence rate.

\section{Concluding remarks}

We have proposed and analyzed a cut finite element method for a rectangular plate element, allowing for curved boundaries. The analysis shows that the method is optimally order convergent and stable. Two different approximations of the boundary have been tested, a standard cubic interpolation of the exact boundary and a cubic spline approximation leading to a continuously differentiable approximation of the boundary. Numerical results and theory indicate that the continuity of the boundary approximation is not 


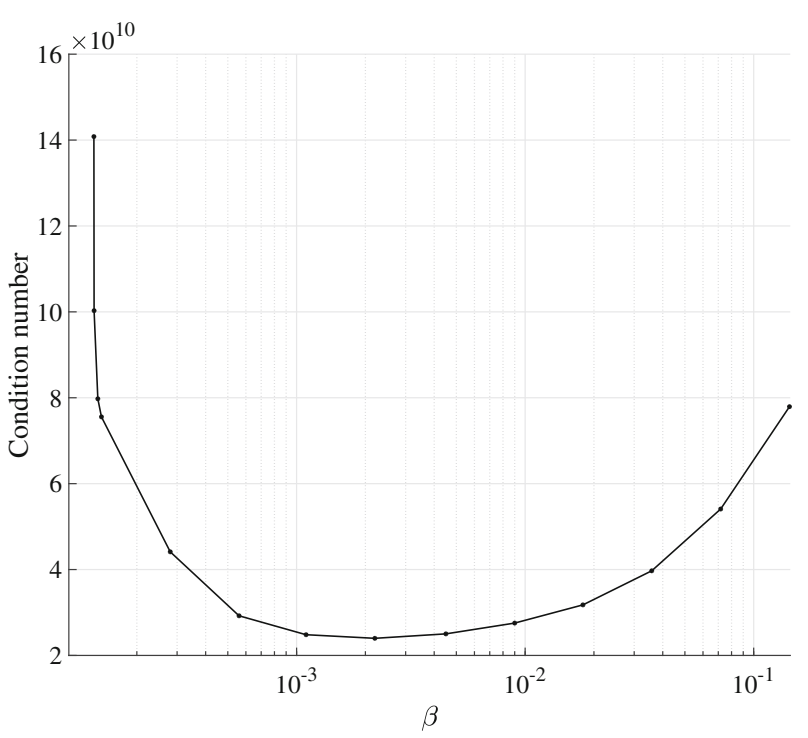

Fig. 8 Variation of the condition number as a function of $\beta$

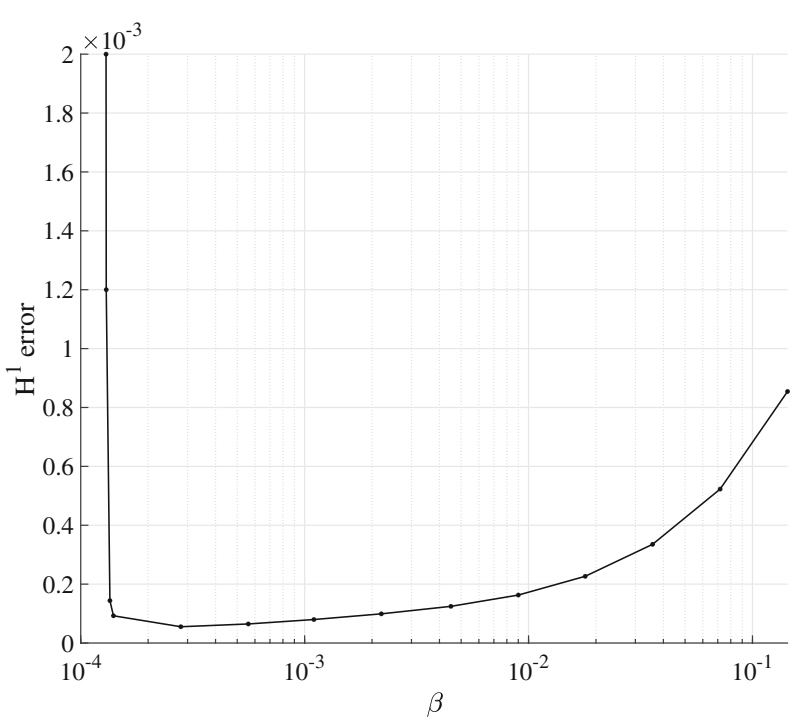

Fig. 9 Variation of the $H^{1}$ error as a function of $\beta$

crucial. With our method, the simple rectangular $C^{1}$ element has greatly increased its practical applicability.

\section{Funding}

Open access funding provided by Jönköping University. Funding was provided by Stiftelsen för Strategisk Forskning (AM13-0029), Vetenskapsrådet (2013-4708, 2017-03911, 2018-05262), Engineering and Physical Sciences Research Council (EP/P01576X/1) and Swedish Research Programme Essence.

\section{Author details}

${ }^{1}$ Department of Mathematics, University College London, London WC1E 6BT, UK, ${ }^{2}$ Department of Mechanical

Engineering, Jönköping University, 55111 Jönköping, Sweden, ${ }^{3}$ Department of Mathematics and Mathematical Statistics, Umeå University, 90187 Umeå, Sweden. 


\section{Appendix: Some inequalities}

Let $\omega \subset \partial \Omega$ and define the cylindrical tubular neighborhood

$$
U_{\delta}(\omega)=\left\{x \in U_{\delta}(\partial \Omega): p(x) \in \omega\right\} \quad \delta \in\left(0, \delta_{0}\right]
$$

Let $\Gamma_{1}$ and $\Gamma_{2}$ be two surface segments in $U_{\delta}(\omega)$ with unit normals $n_{i}$, such that the closest point mapping $p: \Gamma_{i} \rightarrow \omega$ is a bijection with inverse $p_{i}^{-1}: \omega \rightarrow \Gamma_{i}$ and there is constant such that

$$
1 \lesssim \min _{x \in \Gamma_{i}}\left|n_{i}(x) \cdot n(x)\right|
$$

where $n(x)=n \circ p(x)$. We can then define a bijection $q: \Gamma_{1} \rightarrow \Gamma_{2}$ by $q=p_{2}^{-1} p$. For each $x \in \Gamma_{1}$ let $I_{x}$ be the line segment with endpoints $x \in \Gamma_{1}$ and $q(x) \in \Gamma_{2}$. We then have

$$
v(x)=v(q(x))+\int_{I_{x}} \nabla_{n} v d s
$$

where we integrate along $I$ from $q(x)$ to $x$. Using the Cauchy Schwarz inequality we get

$$
\begin{aligned}
v^{2}(x) & \lesssim v^{2}(q(x))+\left(\int_{I_{x}} \nabla_{n} v\right)^{2} \lesssim v^{2}(q(x))+\int_{I_{x}} 1 \int_{I_{x}}\left|\nabla_{n} v\right|^{2} \\
& \lesssim v^{2}(q(x))+\delta \int_{I_{x}}\left|\nabla_{n} v\right|^{2}
\end{aligned}
$$

Integrating over $\Gamma_{1}$,

$$
\begin{aligned}
\int_{\Gamma_{1}} v^{2}(x) & \lesssim \int_{\Gamma_{1}} v^{2}(q(x))+\delta \int_{\Gamma_{1}} \int_{I_{x}}\left|\nabla_{n} v\right|^{2} \\
& \lesssim \int_{\Gamma_{2}} v^{2}\left(y_{2}\right)+\int_{S}\left|\nabla_{n} v\right|^{2}
\end{aligned}
$$

Here $S=\cup_{x \in \Gamma_{1}} I_{x}$ is the domain between the surfaces $\Gamma_{1}$ and $\Gamma_{2}$, and we changed coordinates to integration over $\Gamma_{2}$ and $S$ equipped with Euclidian measure. We conclude that

$$
\|v\|_{\Gamma_{1}}^{2} \lesssim\|v\|_{\Gamma_{2}}^{2}+\delta\|\nabla v\|_{S}^{2}
$$

In applications, it is of convenient to simply replace $S$ by the larger domain $U_{\delta}(\omega)$.

Typical applications include taking $\Gamma_{2}=\omega=\partial \Omega$ and $\Gamma_{1}=\partial \Omega_{h}$, which gives

$$
\|v\|_{\partial \Omega_{h}}^{2} \lesssim\|v\|_{\partial \Omega}^{2}+\delta\|\nabla v\|_{S}^{2}
$$

For $v \in V_{h}$ we have $S \subset O_{h}=\cup_{T \in \mathcal{T}_{h}} T$ since we assume the $\Omega \cup \Omega_{h} \subset O_{h}$ and in particular $S \subset U_{\delta}(\partial \Omega) \cap O_{h}$ and thus we get

$$
\|v\|_{\partial \Omega_{h}}^{2} \lesssim\|v\|_{\partial \Omega}^{2}+\delta\|\nabla v\|_{U_{\delta}(\partial \Omega) \cap O_{h}}^{2}
$$

For $v \in H^{1}(\Omega)$, with extension to $\mathbb{R}^{2}$ also denoted by $v$, we have $S \subset U_{\delta}(\partial \Omega)$ and we get

$$
\|v\|_{\partial \Omega_{h}}^{2} \lesssim\|v\|_{\partial \Omega}^{2}+\delta\|\nabla v\|_{U_{\delta}(\partial \Omega) \cap O_{h}}^{2}
$$

In this paper we have recall that $\partial \Omega_{h} \subset U_{\delta}(\partial \Omega)$, with $\delta \sim h^{4}$, see (10). 


\section{References}

1. Argyris JH, Fried I, Scharpf DW. The tuba family of plate elements for the matrix displacement method. Aeronaut J Roy Aeronaut Soc. 1968;72(692):701-9.

2. Arnold DN, Falk RS. A uniformly accurate finite element method for the Reissner-Mindlin plate. SIAM J Numer Anal. 1989;26(6):1276-90.

3. Babuška I, Pitkäranta J. The plate paradox for hard and soft simple support. SIAM J Math Anal. 1990:21(3):551-76.

4. Beirão da Veiga L, Niiranen J, Stenberg R. A family of $C^{0}$ finite elements for Kirchhoff plates. II. Numerical results. Comput Methods Appl Mech Eng. 2008;197(21-24):1850-64.

5. Bernadou M, Boisserie J-M. Curved finite elements of class $C^{1}$ : implementation and numerical experiments. I. Construction and numerical tests of the interpolation properties. Comput Methods Appl Mech Eng. 1993;106(1-2):229-69.

6. Bogner FK, Fox RL, Schmit LA. The generation of interelement compatible stiffness and mass matrices by the use of interpolation formulae. In: Proc. Conf. Matrix Methods in Struct. Mech., AirForce Inst. of Tech., Wright Patterson AF Base, Ohio. 1965. p. 397-444.

7. Brenner SC, Scott LR. The mathematical theory of finite element methods, volume 15 of Texts in Applied Mathematics. 3rd ed. New York: Springer; 2008

8. Brenner SC, Sung L-Y. C $C^{0}$ interior penalty methods for fourth order elliptic boundary value problems on polygonal domains. J Sci Comput. 2005:22(23):83-118.

9. Burman E, Hansbo P, Larson MG. A cut finite element method with boundary value correction. Math Comp. 2018;87(310):633-57.

10. Clough RW, Tocher JL. Finite element stiffness matrices for analysis of plate bending. In: Matrix methods in structural mechanics, number AFFDL-TR-66-80. 1966. p. 515-45.

11. Di Pietro DA, Ern A. Mathematical aspects of discontinuous Galerkin methods, volume 69 of Mathématiques \& Applications (Berlin) [Mathematics \& Applications]. Heidelberg: Springer; 2012

12. Durán R, Liberman E. On mixed finite element methods for the Reissner-Mindlin plate model. Math Comp. 1992;58(198):561-73.

13. Ern A, Guermond J-L. Theory and practice of finite elements, volume 159 of Applied Mathematical Sciences. New York: Springer; 2004

14. Gilbarg D, Trudinger NS. Elliptic partial differential equations of second order. Classics in Mathematics. Berlin: Springer: 2001. Reprint of the 1998 edition.

15. Hansbo P, Larson MG. A discontinuous Galerkin method for the plate equation. Calcolo. 2002;39(1):41-59.

16. Hansbo P, Larson MG, Larsson K. Cut finite element methods for linear elasticity problems. In: Geometrically unfitted finite element methods and applications, volume 121 of Lect. Notes Comput. Sci. Eng.. Cham: Springer; 2017. p. 25-63.

17. Huang J, Huang X. A hybridizable discontinuous Galerkin method for Kirchhoff plates. J Sci Comput. 2019;78(1):290320

18. Huston R, Josephs H. Practical stress analysis in engineering design. Boca Raton: CRC Press; 2008.

19. Massing A, Larson MG, Logg A, Rognes ME. A stabilized Nitsche fictitious domain method for the Stokes problem. J Sci Comput. 2014:61(3):604-28.

20. Nitsche JA. Über ein Variationsprinzip zur Lösung von Dirichlet-Problemen bei Verwendung von Teilräumen, die keinen Randbedingungen unterworfen sind. Abh Math Univ Hamburg. 1971;36:9-15.

21. Stein EM. Singular integrals and differentiability properties of functions. Princeton Mathematical Series, No. 30 Princeton: Princeton University Press; 1970

22. Wells GN, Dung NT. A C $C^{0}$ discontinuous Galerkin formulation for Kirchhoff plates. Comput Methods Appl Mech Eng 2007;196(35-36):3370-80.

23. Zhang S. On the full $C_{1}-Q_{k}$ finite element spaces on rectangles and cuboids. Adv Appl Math Mech. 2010;2(6):701-21.

24. Zienkiewicz OC, Taylor RL. The finite element method. Vol. 2: solid mechaincs. 5th ed. Oxford: Butterworth-Heinemann; 2000.

25. Hansbo A, Hansbo P, Larson MG. A finite element method on composite grids based on Nitsche's method. ESAIM: Math. Model. Numer. Anal. 2003:37(3):495-514.

\section{Publisher's Note}

Springer Nature remains neutral with regard to jurisdictional claims in published maps and institutional affiliations. 